\title{
A Probabilistic Neural Network for Attribute Selection in Stereovision Matching
}

\author{
G. Pajares and J. M. de la Cruz \\ Dpto. Arquitectura de Computadores y Automática, Facultad de CC. Físicas, Universidad Complutense, Madrid, Spain
}

The key step in stereovision is image matching. This is carried out on the basis of selecting features, edge points, edge segments, regions, corners, etc. Once the features have been selected, a set of attributes (properties) for matching is chosen. This is a key issue in stereovision matching. This paper presents an approach for attribute selection in stereovision matching tasks based on a Probabilistic Neural Network, which allows the computation of a mean vector and a covariance matrix from which the relative importance of attributes for matching and the attribute interdependence can be derived. This is possible because the matching problem focuses on a pattern classification problem. The performance of the method is verified with a set of stereovision images and the results contrasted with a classical attribute selection method and also with the relevance concept.

Keywords: Feature selection; Matching; Probabilistic neural networks; Stereovision

\section{Introduction}

The key step in stereovision is image matching, namely, the process of identifying the corresponding points in two images that are generated by the same physical point in a 3D scene. The stereo correspondence problem can be defined in terms of finding pairs of true matches that satisfy three competing constraints: similarity, smoothness and uniqueness [1]. The similarity constraint is associa-

Correspondence and offprint requests to: Facultad de CC. Físicas, Universidad Complutense, 28040 Madrid, Spain. Email: pajares@dacya.ucm.es ted with a local matching process, where a minimum difference attribute (properties of features) criterion is applied. The results computed in the local process are later used by a global matching process where other constraints such as smoothness [1], minimum differential disparity [2] and figural continuity [3] are imposed. A good choice of local matching strategy is the key to good results in the global matching process.

This paper is devoted solely to the local matching process, and presents an approach where the stereovision local matching problem is formulated as a pattern classification problem following a Probabilistic Neural Network (PNN) strategy [4], from which the relative importance of attributes for matching is derived.

Once the features have been selected, the main problem is to choose the set of attributes to be computed, i.e. which of the available attributes should be used for the local matching. This is known as 'attribute selection' in stereovision matching. Attribute selection is non-trivial for a number of reasons [5]. First, the attributes are seldom entirely independent. There may be redundancy, where certain variables are correlated and it is perhaps unnecessary to include all of them in the matching metric for computing the similarity between features. Hence, in some cases, it may be beneficial to discard variables that have some low level of irrelevant information.

Section 2 discusses the choice of this approach and its research motivation. Section 3 describes the experiments. In particular, in Sections 3.3.1 and 3.3.2, comparative analysis are carried out against a classical attribute selection method and according to the relevance concept, respectively. The relevance concept is described in such sub-section. Finally, Section 4 outlines the conclusions. 


\section{Probabilistic Neural Networks in Stereovision Matching}

PNNs are used for general classification problems [6,7], for feature selection [5], and also for stereovision matching $[4,8,9]$. Therefore, we have all the basic structure for our purpose. Indeed, in Specht [6] and Traven [7], the general approach for classification problems is described, where the main goal is to assign cases to one of a number of discrete classes. The output of the model is an estimate of the class membership probabilities. Hunter [5] uses the PNN to estimate the probability density function and the prior probabilities in a straightforward fashion. The probabilities are estimated by using non-parametric techniques, and then the PNN is used to evaluate a variable subset. Finally, a parametric technique is used to compute the matching probability $[4,8,9]$.

Given a stereo-pair of features where one feature comes from the left image and the other from the right image, we have $d$ associated attributes for each feature (i.e. two groups of $d$ attributes). With the two groups of attributes we make up two $d$-dimensional vectors $\mathbf{x}_{l}$ and $\mathbf{x}_{r}$, whose $d$ components are the $d$ attribute values of each feature. The sub-indices $l$ and $r$ denote features belonging to the left and right images, respectively. Now, for the given stereo-pair of features, we obtain a $d$-dimensional difference vector of attributes $\mathbf{x}=\mathbf{x}_{l}-\mathbf{x}_{r}$. Hence, an ideal true match is the null representative difference vector $\mathbf{x}$. This is a very important assertion in our approach, as it provides us with a method to derive the relative importance of attributes for matching.

In stereovision matching we are only concerned with the true matches, and the correspondence is based on a metric, usually a minimum distance criterion between attributes of features. Such a metric measures the similarity between pairs of features. We have verified that for true matches the differences in their attributes cluster in a cloud around a centre [8]. We call this cloud the class of true matches. This class is considered a hyper-sphere in $\mathscr{R}^{d}$ with radius $R$. False matches are surrounding the class of true matches, and they can be grouped into classes. Therefore, although there are $N$ classes, only one is the class associated to the true matches. This is the class of interest in our stereovision matching problem.

Now, the goal is to estimate the probability density function associated with the class of true matches. This is carried out through a Finite Mixture of Multivariate Densities approach, based on Maximum Likelihood Estimates.

\subsection{Finite Mixture of Multivariate Densities}

Following Duda and Hart [10], we start by assuming that we know the complete probability structure for the problem with the sole exception of the values of some parameters. To be more specific, we make the following assumptions:

1. The samples come from a set of $N$ known classes $w_{j}$, where $j=1 \ldots N$.

2. The a priori probabilities $P\left(w_{j}\right)$ are also known.

3. The forms of the class-conditional probability densities $p\left(\mathbf{x} \mid w_{j}, \boldsymbol{\delta}_{j}\right)$ are known, $j=1 \ldots N$. We suppose such densities to be normal ones, with the parameter vector given by $\boldsymbol{\delta}_{j}=\left(\boldsymbol{\mu}_{j}, C_{j}\right)$, where $\boldsymbol{\mu}$ and $C$ are the mean vector and the covariance matrix, respectively.

4. All that is unknown are the values for the parameter vector $\boldsymbol{\mu}_{j}, C_{j}$.

Stimuli patterns are assumed to be obtained by selecting a class $w_{j}$ with probability $P\left(w_{j}\right)$, and then selecting an $\mathbf{x}$ according to the probability law $p\left(\mathbf{x} \mid w_{j}, \boldsymbol{\delta}_{j}\right)=N\left(\mathbf{x} \mid w_{j}, \boldsymbol{\mu}_{j}, C_{j}\right)$. Thus, the probability density function for the samples is given by:

$$
p(\mathbf{x} \mid \mathbf{\delta})=\sum_{j=1}^{N} p\left(\mathbf{x} \mid w_{j}, \boldsymbol{\delta}_{j}\right) P\left(w_{j}\right)
$$

A density function of this form is called a mixture density. The conditional density probabilities $p\left(\mathbf{x} \mid w_{j}\right.$, $\boldsymbol{\delta}_{j}$ ) are the component densities, and the a priori probabilities $P\left(w_{j}\right)$ are the mixing parameters [10]. Our basic goal will be to use samples drawn from this mixture density to estimate the unknown parameter vector $\boldsymbol{\delta}$. Once $\boldsymbol{\delta}$ is known, we can break the mixture down into its components. Then, when a difference vector $\boldsymbol{x}$ associated with a pair of features is presented to the system as an input, the pair will be classified as belonging to the class of true matches or to a class of false matches. An unsupervised learning approach is the key step to computing the unknown parameter vector $\boldsymbol{\delta}$. The unsupervised learning process is solved by the Maximum Likelihood approach.

\subsection{Maximum Likelihood Estimates}

Suppose, now, that we are given a set $X=\left\{\mathbf{x}_{l}\right.$, $\left.\ldots, \mathbf{x}_{n}\right\}$ of $n$ unlabelled samples drawn independently from the mixture density (1). The likelihood of the observed samples is by definition the joint density:

$p(X \mid \delta)=\prod_{k=1}^{n} p\left(\boldsymbol{x}_{k} \mid \boldsymbol{\delta}\right)$ 
The maximum likelihood estimate is that value of $\boldsymbol{\delta}=(\boldsymbol{\mu}, C)$ that maximises $p(X \mid \boldsymbol{\delta})$. See Duda and Hart [10] for an exhaustive treatment. Through a stochastic gradient descent solution and after a degree of manipulation, the expressions for $\boldsymbol{\mu}_{j}$ and $C_{j}$ can be obtained from the following recursive expression [7]

$$
\theta_{k+1}=\theta_{k}+\eta_{k+1}\left(\theta\left(\mathbf{x}_{k+1}\right)-\theta_{k}\right)
$$

where $\theta$ means either $\boldsymbol{\mu}_{j}$ or $C_{j}$. This is a learning law, where the new value is obtained by adding to the old value a fraction of the difference between the current sample and the old value. The learning rate $\eta_{k+1}$ is computed as follows:

$$
\eta_{k+1}=\frac{p\left(w \mid \mathbf{x}_{k+1}\right)}{\sum_{i=1}^{k+1} p\left(w \mid \mathbf{x}_{i}\right)}
$$

where $p\left(w \mid \mathbf{x}_{k+1}\right)$ is the probability that once $\mathbf{x}_{k+1}$ is selected it belongs to the class $w$.

The Probability Density Function for each class $w_{j}$ is given by Eq. (5), so given an input vector $\mathbf{x}$ we can compute its probability of association to the class $w_{j}$ through Eq. (5)

$$
\begin{aligned}
& p\left(\mathbf{x} \mid w_{j}, \boldsymbol{\mu}_{j}\right)= \\
& \frac{1}{4 \pi^{2}\left|C_{j}\right|^{0.5}} \exp \left[-\frac{1}{2}\left(\mathbf{x}-\boldsymbol{\mu}_{j}\right)^{t} \mathrm{C}_{j}^{-1}\left(\mathbf{x}-\boldsymbol{\mu}_{j}\right)\right]
\end{aligned}
$$

The expressions in Eqs (3)-(5) can be applied to each class separately. As we are only concerned with the class of true matches, this is the only class of interest to us. Therefore, for simplicity, the class of true matches will be referenced without subscripts. This is also applicable to the parameters associated to this class.

At this moment, our interest is not to compute the matching probability in Eq. (5), but to discover the relative importance of attributes, and also those attributes with redundant information. This allows us to have the option of removing attributes with relatively low importance or with redundant information. Therefore, two goals are to be achieved, namely:

1. To compute the relative importance of the attributes.

2. To obtain the attribute interdependence.

These two goals can be easily reached via from the PNN approach. To be exact, through the examination of the PNN parameters. Indeed once the parameter vector $\boldsymbol{\delta}$ is obtained for the true matches, we use the mean vector $\boldsymbol{\mu}$ to compute the relative importance of the attributes and the covariance matrix $C$ to determine the possible attribute interdependence. This information is very important because we can achieve a better performance, and can also reduce the computational cost, of the process if some attributes can be suppressed according to the information obtained about them. Hence, the motivation for using the proposed method.

\subsection{Computation of the Relative Importance of Attributes for Matching}

As mentioned before, the ideal mean vector for a true stereo pair of features is the null vector. We assume that the initial mean $\boldsymbol{\mu}$ vector value for true matches in Eq. (3) is $\mathbf{0}$. Therefore, as the learning progresses, for each new unlabelled sample the $\boldsymbol{\mu}$ vector is updated according to Eq. (3). After the $n$ unlabelled samples are processed, the $\boldsymbol{\mu}$ vector reaches its final value for the set of samples. Hence, under the above assumptions, we can infer the following: as each component value moves away from the null value, the less is its relative importance for matching. From this assertion, it is easy to derive a method such that the relative importance of the attributes for matching can be quantified, which is achieved as follows. As mentioned before, the initial mean vector $\boldsymbol{\mu}$ for true matches is the null vector. We define a simple metric to measure the distance from 0 for each attribute $k=1, \ldots, d$ as $D_{k}=$ $\left|\mu_{k}\right|+\epsilon$. where $\epsilon \rightarrow 0$ (small quantity to avoid divisions by 0 in Eq. (6), set as 0.01 in this paper); $D=\sum_{k=1}^{d} D_{k}$. Finally, we compute the Relative Importance $(R I)$ factor for each attribute $j$ as follows:

$$
\begin{aligned}
& R I_{j}=\frac{1-D_{j} / D}{\sum_{h=1}^{d}\left(1-D_{h} / D\right)} \\
& =\frac{D-D_{j}}{(d-1) D} ; \quad \text { where } \sum_{j=1}^{d} R I_{j}=1
\end{aligned}
$$

When new samples are available, the $\boldsymbol{\mu}$ vector can be updated and the corresponding $R I_{j}$ factors recomputed.

\subsection{Computation of the Attribute Interdependence}

This is a direct outcome derived from the covariance matrix definition. Indeed, given a $C_{i j}$ element in the covariance matrix for true matches $C$, it is said that the attributes $i, j$ with $i \neq j$ are uncorrelated when $c_{i j}$ is zero. This means that there is no redundancy between attributes $i, j$, otherwise an underlying inter- 
dependence is established between these two attributes [11].

Initially, we assume pairs of attributes to be uncorrelated, and the covariance matrix is chosen as the identity matrix so that the updating process through Eq. (3) starts with the covariance matrix as a $d \times d$ identity matrix. As the learning progresses, the covariance matrix is updated and the elements off the principal diagonal move away from zero. As these elements increase their values, a greater correlation is established between the associated attributes.

Taking into account the relative importance and the correlation between attributes, we can determine when the contribution of a given attribute is not relevant for matching, and such an attribute can be removed.

\section{Performance}

To assess the validity of the method, we have chosen edge segments as features and have selected four attributes for matching, namely: gradient magnitude, gradient direction, Laplacian and variance. The gradient magnitude and the Laplacian are chosen intentionally, as they are both derivative operators and therefore a correlation is likely.

We first give some details about the edge segment extraction and the attribute computation for facility. Following this, we present the experimental results.

\subsection{Feature Extraction and Attribute Computation}

The contour edges in both images are extracted using the Laplacian of the Gaussian filter in accordance with the zero-crossing criterion [12]. For each zero-crossing in a given image, its gradient vector (magnitude and direction) as in Leu and Yau [13], Laplacian and variance as in Krotkov [14] are computed from the grey levels of a central pixel and its eight immediate neighbours. To find the gradient magnitude of the central pixel, we compare the grey level differences from the four pairs of opposite pixels in the 8-neighbourhood; the largest difference is taken as the gradient magnitude. The gradient direction of the central pixel is the one the eight principal directions whose opposite pixels yield the largest grey level difference, and also points in the direction which the pixel grey level is increasing. A chain-code with eight principal directions allows the normalisation of the gradient direction. Once the zero-crossings are detected, we use the following two algorithms to extract the edge segments or features:

(a) adjacent zero-crossings are connected if their corresponding differences in gradient magnitude and gradient direction don't surpass the quantities of $\pm 20 \%$ and $\pm 45^{\circ}$, respectively [15];

(b) each detected contour according to the preceding algorithm is approximated by a series of piecewise linear line segments [16].

Hence, we have built edge segments made up of a certain number of zero-crossings. As stated before, for each zero-crossing we have computed four attributes (magnitude and direction gradient, Laplacian and variance). We consider the four attributes for all zero-crossings belonging to a given edge segment, and for each attribute an average value is finally obtained. All average attribute values are scaled, so that they fall within the same range. These four averaged values are the associated attributes of the given edge segment. Moreover, each edge segment is identified by its initial and final pixel coordinates, its length and its label.

A pair of edge segments is a potential match if the two segments verify the following three initial conditions: (1) the absolute value of the difference in the gradient vector direction between the two segments is below a specific threshold, set at $20^{\circ}$; (2) the absolute value of the difference in the gradient magnitude is also below a fixed threshold, set at 10 ; (3) the overlap rate surpasses a given value, set at 0.8 . The 'overlapping' is a concept introduced in Medioni and Nevatia [2] as follows: two segments overlap if by sliding one of them in a direction parallel to the epipolar line, i.e. to an horizontal line, they would intersect. We apply this concept to a stereo pair of edge segments. This stereo pair is made up of an edge segment of the left image and an edge segment of the right image. The axes of the two cameras of the stereo image system are previously aligned, so that the images are also aligned, i.e. the vertical positions of the images of any point in the scene are approximately similar in both images.

Therefore, given a stereo pair of edge segments, where an edge segment comes from the left image and the other from the right image, we have four associated attributes for each edge segment (i.e. two groups of four attributes). With the two groups of attributes we make up two four-dimensional vectors $\mathbf{x}_{l}$ and $\mathbf{x}_{r}$ whose four components are the four averaged attribute values of each edge segment. The superscripts $l$ and $r$ denote edge segments belonging to the left and right images, respectively. Now, for the given stereo-pair of edge segments, we obtain a 
four-dimensional difference vector of attributes $\mathbf{x}=$ $\mathbf{x}_{l}-\mathbf{x}_{r}=\left\{x_{m}, x_{d}, x_{p}, x_{v}\right\}$. The components of $\mathbf{x}$ are the corresponding differences for module and direction gradient, Laplacian and variance, respectively.

\subsection{Experimental Results}

We have used 47 pairs of stereo images coming from an indoor environment where edge segments are abundant. A representative stereo pair is shown in the Figs 1 (a)-(d). This is a full representative set of the environment of interest. This environment is where our mobile robot, equipped with our stereovision system, navigates.

A number of 27 stereo images is used to compute the parameter vector $\boldsymbol{\delta}=(\boldsymbol{\mu}, C)$ for the class of true matches with 1124 pairs of edge segments. With this set of samples we obtain the values given in Eq. (7) for the mean vector and the covariance matrix,

$$
\begin{aligned}
\boldsymbol{\mu} & =\{0.543,-0.142,0.924,0.948\} \\
C & =\left[\begin{array}{llll}
0.990 & 0.011 & 0.801 & 0.015 \\
0.011 & 0.965 & 0.111 & 0.026 \\
0.801 & 0.111 & 1.200 & 0.035 \\
0.015 & 0.026 & 0.035 & 1.178
\end{array}\right]
\end{aligned}
$$

Next, with these two parameters we use Eq. (5) to match 800 pairs of edge segments extracted from 20 stereo images. The success rate reaches $94 \%$.

From the results obtained in Eq. (7) for the mean vector, we now compute the relative importance factors $R I_{j}$, through Eq. (6). These factors are then converted to percentage values, with the following final results: $26.25 \%, 31.48 \%, 21.29 \%$ and $20.98 \%$ for gradient magnitude, gradient direction, Laplacian and variance, respectively. Taking into account the two derivative attributes, i.e. gradient magnitude (first attribute) and Laplacian (third attribute), it is clear that the Laplacian has less importance than the gradient magnitude. Moreover, from the covariance matrix values, it is also clear that the gradient magnitude and Laplacian have a high correlation coefficient compared with remaining values. Hence, we choose to remove the Laplacian attribute, and repeat the process with the remaining three attributes, i.e. the computation of a new parameters vector $\boldsymbol{\delta}=(\boldsymbol{\mu}, C)$, where the four dimensional space is reduced to a three-dimensional one. After this, and once again using Eq. (6) the success rate obtained is still $94 \%$. The computational time has been reduced by $18 \%$ with respect to the previous one when the Laplacian was used. This proves the Laplacian attribute to be unnecessary from this view point, and for the proposed matching purpose. Its discriminatory properties can be subsumed by the gradient magnitude attribute.

\subsection{Comparative Analysis}

Apart from the results obtained in the previous section, we have selected two different methods to assess the validity of our approach.

3.3.1. Learning and feature selection. This is the method described in Leu et al. [17] for stereovision matching. It uses points as features instead of the edge segments used in this paper, and proposes the following possible attributes associated with each point $(x, y)$ : intensity, Gradient (magnitude and orientation), Laplacian and Curvature. The goal is to establish the correspondence between a template point $\left(x_{p}, y_{p}\right)$ and a matching point $\left(x_{c}, y_{c}\right)$. The central idea is to find a subset of these attributes for $\left(x_{p}, y_{p}\right)$ that will uniquely define the point. As in this paper, they compute the importance of attributes for the matching. Although the features used are different, they also conclude that the Laplacian attribute has

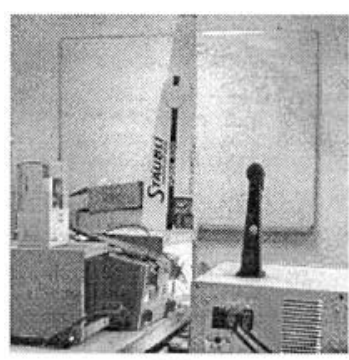

(a)

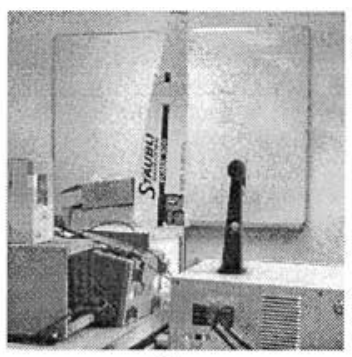

(b)

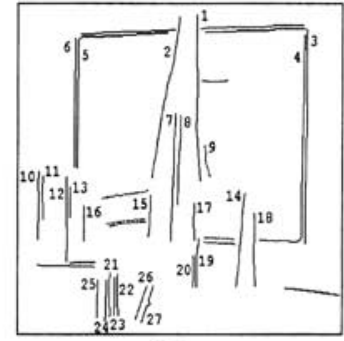

(c)

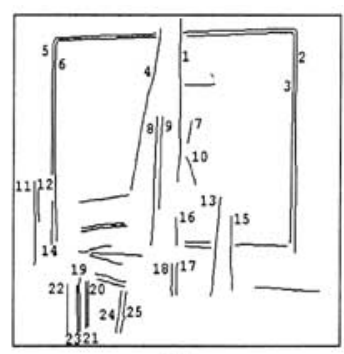

(d)

Fig. 1. (a) and (b) original left and right stereo images respectively; (c) and (d) edge segments for the left and right stereo images respectively). 
less relative importance than the gradient magnitude. This is the same conclusion as that reached in this work.

3.3.2. Feature subset selection based on relevance. This is the axiomatic approach described in Wang et al. [18] based on the relevance concept. To understand the proposed method we give some details taken from Wang et al. [18]. Relevance is a common sense notion, we say object $X$ is relevant to object $Y$ if knowing $X$ happening would change the likelihood of $Y$. In information theoretic terms, the relevance of one variable to another (target) variable is interpreted as the mutual information between the two variables relative to the entropy of the target variable, or in other words, the relative reduction of entropy (uncertainty) of one variable due to the knowledge of another. The bigger the reduction the higher the relevance. Formally, the variable relevance of $X$ to $Y$ given $Z$, denoted $r(X ; Y \mid Z)$, is defined as

$$
r(X ; Y \mid Z)=\frac{I(X ; Y \mid Z)}{H(Y \mid Z)}
$$

where $X, Y, Z$ are random variables with a joint probability distribution; $H(Y \mid Z)$ the entropy of $Y$ given $Z$ and $I(X ; Y \mid Z)$ the mutual information. According to Gonzalez and Woods [19], the mutual information is given by $I(X ; Y \mid Z)=\log \frac{1}{p(X ; Y \mid Z)}=$ $-\log p(X ; Y \mid Z)$ and the entropy by $H(X)=-\Sigma_{x} p(x)$ $\log p(x)$. The information content of a message is inversely related to its probability. If $p()=$.1 , the event always occurs and no information is attributed to it. The average information content per message of a set of messages is its entropy.

Our interest is centred on the computation of the relevance between the variables $\left\{x_{m}, \mathrm{x}_{d}, \mathrm{x}_{p}, \mathrm{x}_{v}\right\}$, specifically the relevance of $x_{i}$ to the target $x_{j}$ given the other two variables. So, we can define $X=$ $\left\{x_{i}\right\}, Y=\left\{x_{j}\right\}$ and $Z=\left\{x_{h}, \mathrm{x}_{k}\right\}$, where $i \neq j \neq h$ $\neq k$. We introduce a slight modification, instead of computing the relevance between two variables, we compute the relevance of $x_{i}$ to $x_{j}, \mathrm{x}_{h}, \mathrm{x}_{k}$ as follows:

$$
\begin{aligned}
& r\left(x_{i}\right)=\frac{I\left(x_{i}, x_{j}, x_{h}, x_{k}\right)}{H\left(x_{j}, x_{h}, x_{k}\right)} \\
& =\frac{-\log \bar{p}(\mathbf{x})}{\sum_{g=j, h, k} \bar{p}\left(x_{g}\right) \log \bar{p}\left(x_{g}\right)}
\end{aligned}
$$

where $\mathbf{x}=\left\{x_{m}, \mathrm{x}_{d}, \mathrm{x}_{p}, \mathrm{x}_{v}\right\} ; \bar{p}($.$) is the average$ probability computed, taking into account the probabilities $p($.$) over all the pairs of edge segments$ classified as true from the 800 tested pairs. $p(\mathbf{x})$ is computed exactly as in Eq. (5) and $p\left(x_{g}\right)$ is also computed as in Eq. (5), but with $\mathbf{x}=\left\{x_{g}\right\}$, i.e. considering a one-dimensional distribution. In this last case, the training and classification processes are repeated for each variable $x_{\mathrm{g}}$.

Table 1 shows the variable relevance of each variable $x_{i}$ to the remaining three variables. Although the differences are not significant, we still can see how the relevance of the variable $x_{l}$ (Laplacian) is the greatest. This means that its entropy is reduced due to the knowledge of the other three. This is in accordance with the results obtained previously.

\section{Concluding Remarks}

The PNN has proven to be a suitable method for attribute selection in stereovision matching. Indeed, it allows us to discover if there is redundant information in the attributes used and hence to remove the unnecessary attribute or attributes.

We have removed the attribute associated with the Laplacian, and the percentage of successes does not vary with regard to the use of the four original attributes. This does not mean that an irrelevant attribute must be removed in a mandatory fashion. We only offer this possibility to improve the computational time, because we consider that this is a key issue for real time matching processes. This is also the underlying idea in Leu et al. [17]. Nevertheless, in some experiments a redundant attribute still retains a few discriminatory properties, or is useful for other purposes, and it should indeed be kept.

Although the relevance study is not conclusive, due to the small difference values, we provide with its introduction a guide, and the possibility of it been used in other applications.

Also, although our experiments have been carried out in an indoor environment, the method is applicable to other types of scenario, such as outdoor spaces where the types of feature are different. This is because the method discriminates between attributes.

We have selected two attributes (gradient magnitude and Laplacian) whose interdependence was clear a priori, as they are derivative operators. Nevertheless, sometimes this is unclear, and the

Table 1. Relevance of each variable to the other three variables

\begin{tabular}{lllll}
\hline & $\mathbf{x m}$ & $\mathbf{x d}$ & $\mathbf{x p}$ & $\mathbf{x v}$ \\
\hline$r_{i} \times 10^{3}$ & 62.1 & 60.1 & 64.5 & 63.3 \\
\hline
\end{tabular}


method of this paper provides the necessary criterion to take a decision about attribute redundancy. This is the case when the features are regions and the attributes are the set of seven $\mathrm{Hu}$ invariant moments by example.

In earlier work [20] we have carried out comparative analyses between different local learning matching strategies, and the non-parametric Parzen's window estimation approach is compared with the PNN proposed in this paper [21]. We have also included both PNN and Parzen's window in global matching strategies [21] as a mapping of the similarity constraint competing with the smoothness and uniqueness global matching constraints.

Finally, we conclude that the method has global validity, and it is suitable for attribute selection in stereovision image matching.

Acknowledgements. Part of the work has been performed under project CICYT TAP94-0832-C0201. The constructive recommendations provided by the reviewers are also gratefully acknowledged.

\section{References}

1. Marr D, Poggio TA (1979) Computational theory of human stereovision. Proc Royal Society of London B207: 301-328

2. Medioni G, Nevatia R (1985) Segment based stereo matching. Computer Vision, Graphics, and Image Processing 31: 2-18

3. Pollard SB, Mayhew JEW, Frisby JP (1981) PMF: A stereo correspondence algorithm using a disparity gradient limit. Perception 14: 449-470

4. Cruz JM, Pajares G, Aranda J (1995) A neural network approach to the stereovision correspondence problem by unsupervised learning. Neural Networks 8(5): 805-813

5. Hunter A (2000) Feature selection using probabilistic neural networks. Neural Computing and Applications 9: $124-132$

6. Specht DF (1990) Probabilistic neural networks. Neural Networks 3(1): 109-118
7. Traven HGC (1991) A neural network approach to statistical pattern classification by semiparametric estimation of probability density function. IEEE Trans Neural Networks 2: 366-377

8. Pajares G (1995) Estrategia de Solución al Problema de la Correspondencia en Vision Estereoscópica por la Jerarquia Metodológica y la Integración de Criterios, $\mathrm{PhD}$ thesis, Dpto. Informática y Automática, Facultad Ciencias UNED: Madrid

9. Pajares G, Cruz JM, López JA (1998) Pattern recognition learning applied to stereovision matching. In: Amin A, Dori D, Pudil P, Freeman H, editors, Advances in Pattern Recognition. Springer-Verlag, Berlin, 997-1004

10. Duda RO, Hart PE, Stork, DG (2001) Pattern Classification and Scene Analysis. Wiley, New York

11. Maravall D (1993) Reconocimiento de Formas y Visión Artificial. RA-MA, Madrid

12. Huertas A, Medioni G (1986) Detection of intensity changes with subpixel accuracy using Laplacian-Gaussian masks. IEEE Trans Pattern Anal Machine Intell 8(5): 651-664

13. Leu JG, Yau HL (1991) Detecting the dislocations in metal crystals from microscopic images. Pattern Recognition 24(1): 41-56

14. Krotkov EP (1989) Active Computer Vision by Cooperative Focus and Stereo. Springer-Verlag, Berlin

15. Tanaka S, Kak, AC (1990) A rule-based approach to binocular stereopsis. In: Jain RC, Jain AK, editors, Analysis and Interpretation of Range Images. SpringerVerlag, Berlin

16. Nevatia R, Babu KR (1980) Linear feature extraction and description. Computer Vision, Graphics and Image Processing 13: 257-269

17. Lew MS, Huang TS, Wong K (1994) Learning and feature selection in stereo matching. IEEE Trans Pattern Anal Machine Intell 16(9): 869-881

18. Wang H, Bell D, Murtagh F (1999) Axiomatic approach to feature subset selection based on relevance. IEEE Trans Pattern Anal Machine Intell 21(3): 271-277

19. Gonzalez RC, Woods RE (1993) Digital Image Processing, Addison-Wesley, Reading, MA

20. Pajares G, Cruz JM (2001) Local stereovision matching through the ADALINE neural network. Pattern Recognition Letters 22: 1457-1473

21. Pajares G, Cruz JM (2002) The non-parametric Parzen's Window in stereo vision matching. IEEE Trans Sys, Man Cybernetics 32(2) 